\title{
Evidence for post-transcriptional regulation of clustered microRNAs in Drosophila
}

\author{
Sergei S Ryazansky ${ }^{1 *}$, Vladimir A Gvozdev ${ }^{1}$ and Eugene Berezikov ${ }^{2,3}$
}

\begin{abstract}
Background: MicroRNAs (miRNA) are short 21-23nt RNAs capable of inhibiting translation of complementary target messenger RNAs. Almost half of D. melanogaster miRNA genes are grouped in genomic clusters.

Results: The peculiarities of the expression of clustered miRNAs were studied using publicly available libraries of sequenced small RNAs from different Drosophila tissues. We have shown that although miRNAs from almost all clusters have similar tissue expression profiles (coordinated clusters), some clusters contain miRNAs with uncoordinated expression profiles. The predicted transcription start sites (TSSs) of such clusters are located upstream of the first miRNA, but no TSSs are found within the clusters. The expression profiles of miR and miR* sequences in uncoordinated clustered miRNAs do not correlate while their profiles from the coordinated clustered miRNAs are similar.
\end{abstract}

Conclusions: The presence of exclusively upstream promoters in miRNA clusters containing uncoordinated miRNAs means that the clusters are transcribed as single transcription units. The difference of tissue expression profiles of uncoordinated miRNAs and the corresponding miRs* suggests a post-transcriptional regulation of their processing or stability.

\section{Background}

MicroRNAs (miRNAs) are short 21-23 nt non-coding RNAs that are processed from one of the arms of hairpin-like 60-100 nt precursor miRNAs (pre-miRNAs). Pre-miRNAs are produced from primary pri-miRNAs transcribed from miRNA genes by RNA polymerase II. Mature miRNAs (miRs) trigger posttranscriptional regulation of the target mRNAs mediated by a specific set of effector proteins [1]. The perfectly complementary miRs induce mRNA degradation in plants, while in animals the partially complementary miRs cause mainly mRNA degradation but also the blocking of translation [2-4]. MiRNA-mediated inhibition of target mRNA translation is considered to be a powerful mechanism of gene expression regulation. Besides the prevalent mature miRs, the minor star molecules $\left(\mathrm{miR}^{*}\right)$ are generated from the opposite arm of the pre-miRNAs and sometimes also capable to inhibit expression of target mRNAs [5,6]. The choice of pre-miRNA strand

\footnotetext{
* Correspondence: ryazansky@img.ras.ru

${ }^{1}$ Institute of Molecular Genetics, Russian Academy of Sciences, Moscow, Russia

Full list of author information is available at the end of the article
}

producing the mature miR is determined by the strands' sequences.

Up to several hundred of miRNA genes are present in eukaryotic genomes, and often miRNAs are located close to each other in a genome, forming genomic clusters [7,8]; for instance, Drosophila melanogaster has at least 176 miRNA genes (miRBase v.16) and almost half of them are clustered. Clustered miRNAs are often coexpressed [9-12] and can jointly regulate functionally related genes, e.g. included in the same signaling pathway $[1,12-14]$. Obviously, the regulation of the expression of individual clustered miRNAs can fine-tune the pathway modulation. Since clusters are considered to be transcribed as single primary pri-miRNA transcripts [7,8,15-17], such regulation can be achieved by posttranscriptional regulation of miRNA maturation [18-21]. In this report we found that several Drosophila miRNA clusters contain miRNAs with the expression profiles different from the profiles of the other miRNAs in the same cluster. Our data argue in favor of a contribution of post-transcriptional rather than transcriptional regulation to the tissue-specific expression of these uncoordinated miRNA clusters. 


\section{Results and discussion Overview of miR clusters}

Here we refer to the grouped miRNA genes as miRNA cluster if they are located not more than $1 \mathrm{~kb}$ apart. A summary of 20 Drosophila miRNA clusters is presented in Additional file 1, Table S1. Using the above criterion, dme-mir-310, -311, -312, -313, -991 and -992 should be related to a single cluster, but the analysis of pair correlation coefficients of their tissue expression profiles (see below) shows that dme-mir-310, -311, -312 and -313 and dme-mir-991, -992 are clearly separated into two clusters, as has been noted before [11]. The 281 cluster, containing a tandem of identical and indistinguishable dme-mir-281-1 and dme-mir-281-2 was excluded from the further expression analyzes. Similarly, identical dmemir-6-1, -6-2, -6-3 from the 6 309 cluster, dme-mir-2a1, $-2 \mathrm{a}-2$ from the $2 \mathrm{a} \sim 2 \mathrm{~b}$ cluster, and dme-mir-983-1, -983-2 from the 983 984 cluster were considered as a single miRNA within each cluster. The subsequent expression analysis showed an adequacy of this simplification. In total, we have examined 19 miRNA clusters.

\section{Some clustered miRNAs have uncoordinated expression profiles}

To determine the miRNA expression profiles, we analyzed 16 million reads of sequenced small RNAs from 9 publicly available libraries prepared from heads, bodies, testes, ovaries, embryos and S2 cells (Additional file 1,
Tables S2 and S3). The coordinated expression of clustered miRNAs has been described earlier in human, mouse and fruit fly [9-12]. Consistent with these data, we showed that in contrast to miRNAs from different clusters or non-clustered miRNAs, miRNAs from the same cluster tend to be co-expressed in the similar set of tissues $(P$-value $<2 \mathrm{e}-16$, t.test) (Figure $1 \mathrm{a})$. The evaluation of the frequency distributions of the miRNA correlation coefficients of expression profiles also demonstrates that highly correlated miRNAs are mainly related to miRNAs from the same cluster (cl.vs.cl.same, red arrow on the Figure 1b), but not to miRNAs from different clusters. Therefore, expression of miRNA clusters is regulated independently to each another.

While the expression profiles of miRNAs in 14 clusters have a high coefficient of correlation $(r>0.6)$ (Additional file 2), a small fraction of clustered miRNAs have a low correlation coefficient of the expression profiles (cl.vs.cl.same, green arrow on the Figure 1b). Indeed, inspecting the pair correlation coefficient values and heatmaps revealed that 5 clusters (283 12, $275 \sim 305,9 \mathrm{c} \sim 9 \mathrm{~b}, 100 \sim 125,13 \mathrm{~b}-1 \sim 2 \mathrm{c})$ contain miRNAs, the profiles of which differ from the rest of the miRNAs in the same cluster $(r<0.3)$ (Table 1, Additional file 2). The expression levels of uncorrelated miRNAs are sufficiently high and similar to the correlated ones ( $P$-value $=0.27$, t.test, Additional file 1, Table S3), thus the low correlation values of expression profiles are not due to (a)

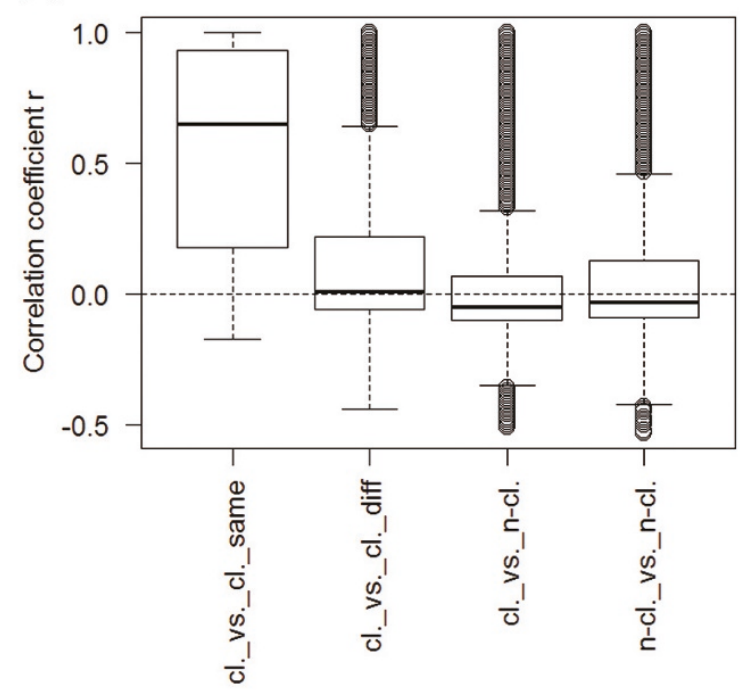

(b)

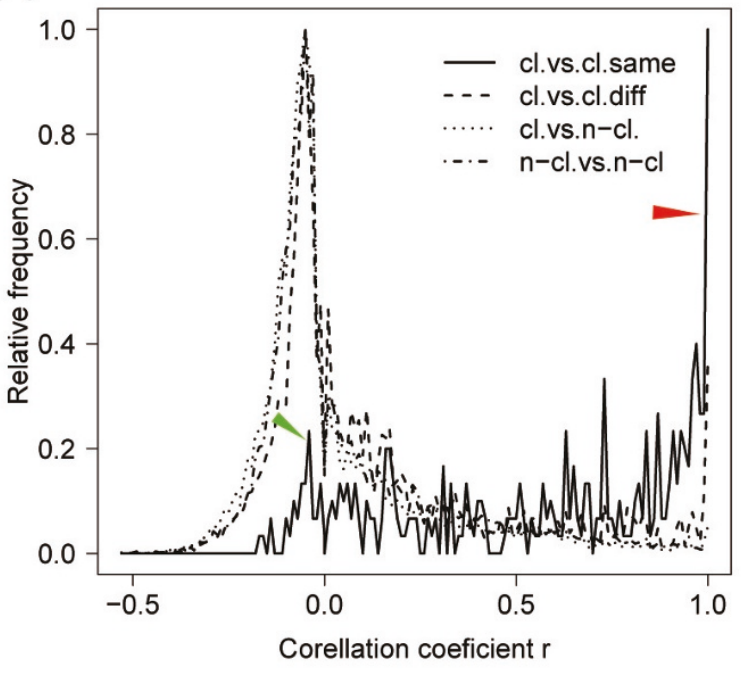

Figure 1 miRNAs with similar expression profiles mainly belong to the same clusters. (a) Pearson correlation coefficients $r$ of expression profiles for miRNA pairs from the same cluster (cl.vs.cl.same), different clusters (cl.vs.cl.diff), clustered and non-clustered miRs (cl.vs.n-cl), and nonclustered miRNAs (n-cl.vs.n-cl.). (b) Frequency distributions of $r$ in the same groups of miRNA pairs. Red and green arrows indicate highly and low correlated fractions of the clustered miRNAs in cl.vs.cl.same group, respectively. Distributions were normalized to the maximum value of frequency in each group. 
Table 1 Clusters with uncorrelated miRNAs

\begin{tabular}{ll}
\hline Cluster name & Uncorrelated miRNA $^{\mathbf{1}}$ \\
\hline $100 \sim 125$ & dme-mir-100 \\
$13 b \sim 2 c$ & dme-mir-13b-1 \\
$275 \sim 305$ & dme-mir-305 \\
$283 \sim 12$ & dme-mir-283 \\
$9 c \sim 9 b$ & dme-mir-306 \\
\hline
\end{tabular}

${ }^{1}$ Uncorrelated miRNAs are characterized by low correlation coefficient with the corresponding $\mathrm{miR}^{*}$; although the 100 125 cluster possibly has two uncorrelated miRNAs, dme-let-7 and dme-mir-100, only dme-mir-100 does not correlate to dme-mir-100* (see text for details).

random noise in miRNA detection. In the 13b 2c cluster a wrong conclusion of a lack of correlation of dmemir-13b-1 to dme-mir-13a and dme-mir-2c can be formally deduced due to the superposition of the expression of the non-clustered paralog dme-mir-13b-2 with identical sequence. The other 4 clusters are likely to have genuinely uncoordinated miRNAs. For instance, the expression profiles of dme-mir-304 and dme-mir-12 in cluster $283 \sim 12$ correlate to one another $(r=0.69)$, but at the same time the profile of dme-mir-283 does not correlate to both dme-mir-304 $(r=0.04)$ and dme$\operatorname{mir}-12(r=0.02)$.

\section{Promoters of miR clusters}

Coordinated expression of clustered miRNAs can be explained by the transcription of a whole cluster as a single polycistronic precursor RNA driven by an upstream promoter, as has been reported earlier [15-17,22,23]. Using RNA-seq and tilling microarrays of RNA samples from 30 fly developmental stages, single pri-miRNAs for 16 non-clustered miRNAs as well as 7 miRNA clusters have been identified [23]. By contrast, uncoordination of the expression profiles of some clustered miRNAs may result from independent transcription of these miRNAs from their own promoters. Moreover, although polycistronic transcript of the uncoordinated 100 125 cluster has been detected in pupae and ecdysone-treated S2 cells [22], it cannot rule out a possibility of the presence the tissue-specific intra-cluster promoters.

To test a possibility of independent transcription of clustered miRs, we have evaluated the positions of their putative promoters in silico. We focused on the search of RNA polymerase II promoters because only a few miRNA genes have RNA polymerase III promoters [24]. It has been shown that transcription start sites (TSSs) of Drosophila pol II promoters associate with the specific pair combinations of sequence motifs [25-27]. In our analysis of cluster promoters we have used a set of TSSs predicted to be located near the detected combinations of such motifs (McPromoter006, [26]). Similar approaches of promoter finding by localization of putative TSSs for human miRNAs have been performed earlier $[28,29]$. Two examples of putative TSSs for coordinated and uncoordinated clusters are shown in Figure 2. The full list of cluster TSSs is presented in Additional file 1, Table S4 and Additional file 3.

The starts of 5'-ends of pri-miRNAs of coordinated 1002 968 and 277 34 clusters [23] are coincided with location of the predicted TSSs within $300 \mathrm{nt}$ range. The 277 34 cluster also has intra-cluster TSSs upstream of dme-mir-34. The next 5 clusters containing miRNAs with coordinated expressions (982 303, 959 964, 994 318, 983 984, 310 313) have one or several TSSs upstream of the first miRNA (Figure $2 \mathrm{a}$ ) but not within the clusters. Another 3 clusters (972 974, 975 977, 978 979) are neighbors and have 2 putative TSSs - the first TSS is located upstream of the 972 974 and the second one is located inside the 972 974 and upstream of the 975 977 and 978-979 clusters. This suggests that the 972 974 cluster may be transcribed from the first TSS and both TSSs may be used to ensure transcription of the other two clusters. The next two coordinated clusters, 998 11 and 2a 2b, are encoded in introns of protein-coding genes and have no predicted TSSs, and coordination of their expression can be explained by the transcription from the host genes promoters. We failed to detect any TSS for the 309 6 cluster, but its single promoter as well as precursor have been identified and experimentally verified earlier $[15,23]$. No nearest TSSs were found for the last 991 992 cluster. Overall, almost all correlated miRNA clusters (12 out of 14) contain only upstream putative pol II promoters ensuring transcription of the whole cluster and coordination of clustered miR expressions (Additional file 3 and Additional file 1, Table S4).

Surprisingly, similarly to the coordinated clusters, the 100 125, 275 305, 9c 9b, 283 12 and 13b 2c clusters containing miRNAs with uncoordinated expression patterns, also have one or several TSSs only upstream of the first miRNA in a cluster (Figure 2b). None of these 5 clusters have any TSS near the internal miRNAs (Additional file 1, Table S4 and Additional file 3).

The distances between miRNAs within clusters usually small (mean $213 \mathrm{nt}$, median $75 \mathrm{nt}$ ), and a lack of putative TSSs can be explained by a low probability of finding them there. To estimate this probability, we calculated an empirical cumulative distribution function (ecdf) of lengths from the starts of fly miRNAs to the nearest upstream putative TSS. Then, the probability $P$ to observe a TSS in a region of the given length $x$ can be determined as $P=\operatorname{ecdf}(x)$. Indeed, the short intermiRNA spaces seem to explain the absence of TSSs within miRNA clusters since their mean $P=0.1$ and median $P=0$ (Additional file 1, Table S5). There are also five $500-900 \mathrm{nt}$ regions with probability $0.33 \leq \mathrm{P} \leq$ 


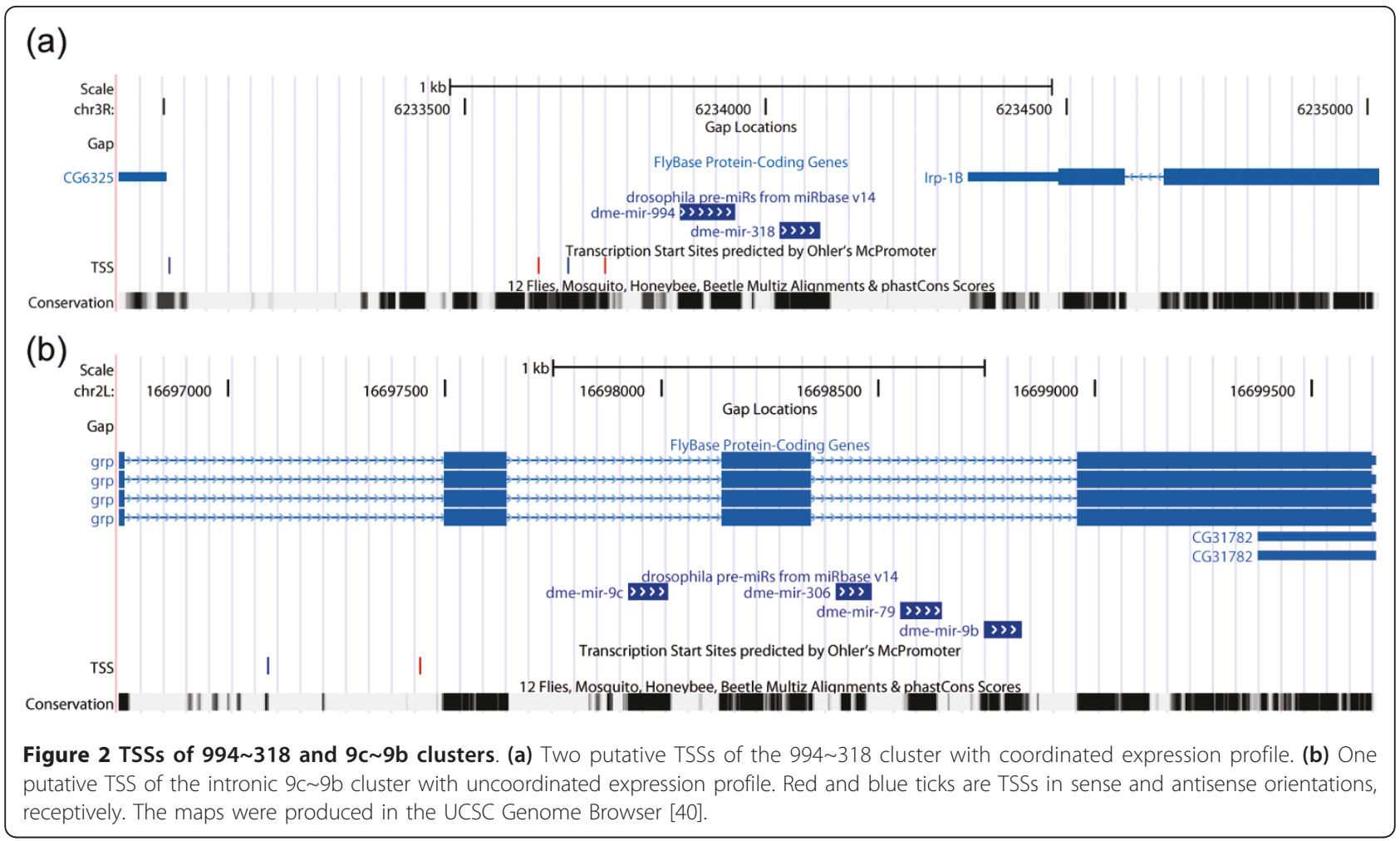

0.44 from coordinated and uncoordinated clusters, one and only one of which (from coordinated 277 34 cluster) contains two TSSs, and this is fully consistent with statistical prediction.

Moreover, to estimate the accuracy of McPromoter in the prediction of miRNA TSSs we checked an enrichment of the TSSs by pol II using the modENCODE ChIP-seq data. We have found that all putative TSSs of 9 miRNA clusters are located in pol II enrichment regions, demonstrating that McPromoter have a very high specificity in TSSs prediction (Additional file 3). The location TSSs of the other clusters near the pol II enriched exons of genes prevents their precise attribution to TSSs. Except for a 277 34, none of the large cluster intra-miRNA regions are enriched by pol II. In addition, there are no significantly conservative regions upstream of uncorrelated miRNAs that could have hinted at the presence of cryptic promoters. (Additional file 3). Therefore, the lack of correlations of the miRNA expression for at least 4 clusters (except the 13b 2c cluster, see above) is unlikely to be explained by independent transcription of the contained miRNA genes.

\section{Comparison of clustered $\mathrm{miR}$ and $\mathrm{miR}^{*}$ expression profiles}

The post-transcriptional regulation of miRNA maturation can be considered as a possible reason of uncoordinated expression of clustered miRs. If alteration of processing or stability happens to miR or its $\mathrm{miR}^{*}$, then their expression profiles may differ, leading to the corresponding low correlation coefficient values. To test this assumption, we determined the correlation coefficients of the expression profiles of clustered miRs and miRs*. Indeed, although expression levels of clustered miRs* are comparable to one another $(P$-value $=0.68$, t.test $)$, the correlation coefficient of expression profiles of almost all $\mathrm{miR} / \mathrm{miR}^{*}$ pairs from each coordinated cluster are high and similar (one exception is the 991 992 cluster, see below), while uncorrelated miRs from each uncoordinated cluster have low correlation coefficients with the corresponding antisense miRs* (Figure 3). For instance, the expression profile of dmemir-306" has a low correlation coefficient with the dmemir-306 profile $(r=0.09)$ and correlates well to all other miRs in the 9c $\sim 9 \mathrm{~b}$ cluster (Figure 3a).

Interestingly, the expression profiles of miRs* of uncorrelated miRs in 283 12 and 277 34 clusters differ not only from their miRs but also from profiles of the rest of miRs in the cluster. Dme-mir-283* correlates neither to dme-mir-283 itself ( $r=0.17)$ nor to dme-mir-304 ( $r=$ $0.1)$ or dme-mir-12 ( $r=0.27)$ (Figure 3b). Similarly, expression of dme-mir-305* from the 275 305 cluster does not correlate both to dme-mir-305 $(r=0.03)$ and dme-mir-275 ( $r=0.16)$ (Figure 3c). This observation can suggest that hairpin processing regulation or independent 


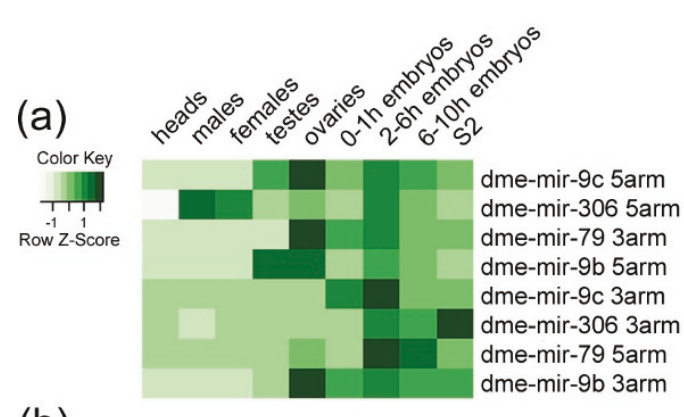

(b)

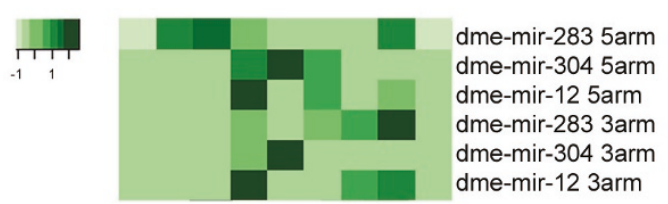

(c)

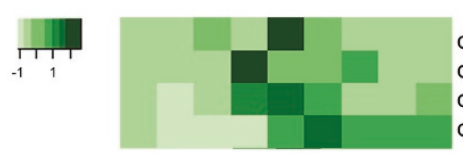

dme-mir-275 3arm dme-mir-305 5arm dme-mir-275 5arm dme-mir-305 3arm

(d)
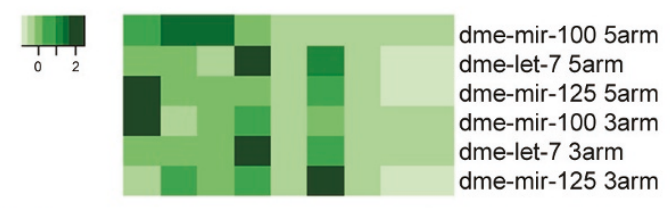

(e)
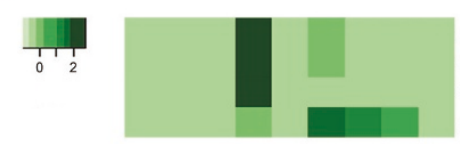

dme-mir-991 3arm dme-mir-992 5arm dme-mir-991 5arm dme-mir-992 3arm

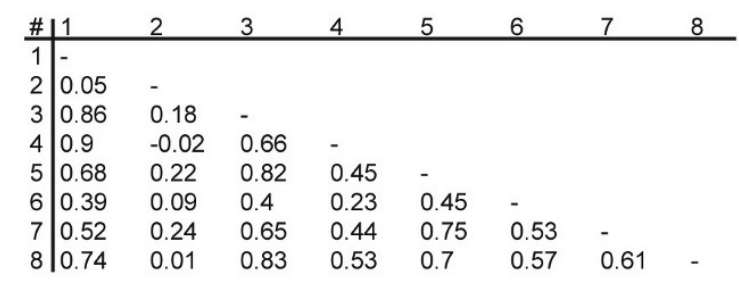

\begin{tabular}{l|llllll}
$\#$ & 1 & 2 & 3 & 4 & 5 & 6 \\
\hline 1 & - & & & & & \\
2 & 0.04 & - & & & & \\
3 & 0.02 & 0.69 & - & & & \\
4 & 0.17 & 0.1 & 0.27 & - & & \\
5 & 0 & 0.74 & 0.17 & -0.15 & - & \\
6 & 0.16 & 0.3 & 0.67 & 0.74 & 0.06 & -
\end{tabular}

\begin{tabular}{l|llll}
$\#$ & 1 & 2 & 3 & 4 \\
\hline 1 & - & & & \\
2 & -0.16 & - & & \\
3 & 0.45 & 0.32 & - & \\
4 & 0.03 & 0.16 & 0.57 & -
\end{tabular}

\begin{tabular}{l|llllll}
$\#$ & 1 & 2 & 3 & 4 & 5 & 6 \\
\hline 1 & - & & & & & \\
2 & 0.27 & - & & & & \\
3 & 0.75 & 0.81 & - & & & \\
4 & 0.29 & 0.49 & 0.48 & - & & \\
5 & 0.35 & 0.93 & 0.76 & 0.53 & - & \\
6 & 0.46 & 0.83 & 0.9 & 0.43 & 0.64 & -
\end{tabular}

\begin{tabular}{l|llll}
$\#$ & 1 & 2 & 3 & 4 \\
\hline 1 & - & & & \\
2 & 0.97 & - & & \\
3 & 0.84 & 0.89 & - & \\
4 & 0.23 & 0.04 & -0.01 & -
\end{tabular}

Figure 3 Expression profiles of clusters containing uncorrelated miRs. The heatmaps (left) and the correlation tables (right) of miRs and miRs* expression profiles from the 9c 9b (a), the 283 12 (b), the 275 305 (c), the 100 125 (d) and the 991 992 (e) clusters. Top miRNAs in the heatmaps and the correlation tables are mature miRs, while the bottom ones are star miRs*.

regulation of stability of not only miRs but also miRs* in some tissue(s) takes place.

Analysis of the pair correlation coefficients of dmemir-100, dme-let-7 and dme-mir-125 for the 100 125 cluster demonstrates the complex nature of post-transcriptional regulation (Figure $3 \mathrm{~d}$ ). It is hard to interpret unambiguously the data but it seems that independent regulation of dme-mir-100 and dme-let-7 is occurred. In human, mouse and nematode Lin 28 protein binds the conserved pre-let-7, inducing hairpin 3'-end uridylation and its degradation [18-21]. Consistently, we propose that in flies the precursor of dme-let-7 is regulated on a level of hairpin stability because expression profiles of dme-let-7 and dme-let-7* are highly similar $(r=0.93)$ but each of them is slightly different from profiles of dme-mir-100 ( $r=0.27$ and 0.35 , respectively) and dmemir-100* ( $r=0.29$ and 0.53 , respectively). A weak correlation of dme-mir-100 and dme-mir-100* ( $r=0.29)$ demonstrates the possibility of dme-mir-100 regulation during its maturation. This is confirmed by finding of the noticeable tissue-specific A-to-I editing of dme-mir100 in male heads and Kc167 cell line [30]. Dme-mir125 and dme-mir- $125 *$ correlate both to dme-mir-100 and dme-let-7, thus complicating the further analysis.

At the same time, miRs and miRs* from almost all clusters with coordinated miR expression profiles have a high correlation coefficient with each other. The only exception is the 991 992 cluster where dme-mir-992* can be considered as a target of post-transcriptional regulation (Figure 3e). In contrast to dme-mir-991\%, the 
expression profile of dme-mir-992* does not correlate to the profiles of dme-mir-991 and dme-mir-992.

One can speculate that clustered miRNAs with uncoordinated expression profiles are transcribed independently and the absence of upstream TSSs is explained by modest sensitivity of the McPromoter program. Since 14 of 24 identified pri-pre-miRNAs [22,23] have one or several TSSs predicted by McPromoter within $300 \mathrm{nt}$ range from their 5' ends (data not shown), a sensitivity for prediction of miRNAs promoters is equal to $60 \%$. This is consisted with previously published data [26] and indicates a possible underrepresentation of the used TSS set. But the lack of correlation of miRs and miRs* strongly suggests their post-transcriptional regulation. Selective post-transcriptional regulation of pre-miRNAs from polycistronic primary pri-miRNAs was reported earlier for the human pre-mir-18a from the miR17 92 cluster [31] and for pre-mir-27a from the miR23 24 [32]. Here we predict that miRs and miRs* from at least 5 fly miR clusters (283 12, 275 305, 9c 9b, 100 125, 991 992) undergo post-transcriptional regulation.

It is necessary to note that analyzed small RNA libraries were prepared by three different laboratories. It was shown that sequencing efficiencies of individual miRNAs may differ for libraries prepared by different ways [33]. Thus, difference in miRNA read counts between libraries may be caused by differential cloning efficiencies rather than reflecting different expression levels. All libraries used in the analysis were prepared according to the same protocol from Pfeffer et al [34] with some lab-specific variations. The main source of small RNA bias in the read frequencies are 5' and 3' adapter ligation steps since three 3'-end nucleotides of acceptor and one $5^{\prime}$ end nucleotide of donor can influence ligation efficiency by T4 RNA ligase 1 [35]. However, the end nucleotide bias for the 3' adapter ligation step can be alleviated by the use of truncated RNA ligase 2, Rnl2(1-249), which has nearly $100 \%$ ligation efficiency regardless of the end nucleotides [36]. Since Rnl2 ligase was used by all three laboratories, it is reasonable to assume that cloning biases due to 3' adapter ligation are not significant. Regarding the $5^{\prime}$ adapter ligation step performed by T4 RNA ligase 1, three 3'-end nucleotides of 5' adapters (acceptor) are highly efficient and identical (-rArArA) for 7 of 9 libraries, thus this is unlikely to be the source of miRNA cloning bias. Only two libraries were prepared using 5' adapter with different 3'-end nucleotides (-rArUrC) that does not introduce a significant impact on total tissue expression profiles. The subsequent reverse transcription and PCR steps also may have some effect on sequencing efficiencies of miRNAs, but it is determined mainly by primary structure of small RNAs itself but not caused by cloning protocols [35]. Although we cannot completely exclude the possibility of cloning artifacts in some analyzed small RNA libraries altering miRNA expression profiles, we suggest that generally our main conclusions are correct. Expanding the set of tissues by libraries from other sources can increase the number of uncorrelated miRNAs and uncoordinated miRNA clusters as well as change the correlation coefficients of above mentioned miRNAs.

To test our conclusions on the independent data, we have conducted the correlation analysis of miRNA expression profiles with the expanded dataset from the recently published libraries [30]. This expanded dataset contains 53 selected libraries with more than 330 million reads (Additional file 1, Tables S6 and S7). In accord with our observations, the expression profiles of dme-mir-100, dme-mir-992, dme-mir-283 and dme-mir306 have low correlation coefficients with profiles of the corresponding miRNAs*. The expression profiles of dme-mir-305, dme-mir-100, dme-mir-992 and dme-mir283 are also weakly correlated to at least one miRNA from their clusters. Thus, although the correlation coefficients for several clustered miRNAs are different, in general the results remain the same.

Several possible mechanisms of miRNA expression regulation have been described, including the modulation of Drosha and Dicer activities, uridylation of premiRNAs and miRs, A-to-I editing of pri- and pre-miRNAs (for review see refs. [37,38]). For dme-let-7 and dme-mir-100 the possible mechanisms of their posttranscriptional regulation are the tissue-specific uridylation and A-to-I editing of their precursors respectively (see above). Interestingly, dme-let-7 as well as clustered coordinated dme-mir-960 and dme-mir-983 were also shown to be A-to-I edited in some tissues [30]. However, the expression levels of these miRNAs expression are very low in these tissues, thus explaining an absence of the influence of editing events on their tissue expression profiles. As the rest of uncoordinated clustered miRNAs seem not to undergo editing in the analyzed tissues, the impact of other mechanisms on the regulation of their expression remains to be revealed.

\section{Conclusions}

In this report we demonstrate that although most Drosophila miRNA clusters are co-expressed in the similar set of tissues, the 283 12, 275 305, 9c 9b, 100 125 and 991 992 clusters contain some miRNAs with different tissue expression profiles compared to other miRNAs from the corresponding clusters. This is unlikely to be explained by independent miRNA transcription because clusters have identifiable putative transcription start sites only upstream of their first miRNA. Most 
probably uncorrelated clustered miRNAs undergo tissue specific post-transcriptional regulation, which is confirmed by a lack of correlation of miR and corresponding $\mathrm{miR}^{*}$ expression profiles.

\section{Methods}

\section{Analysis of small RNA libraries}

The following small RNA libraries were obtained from GEO database and used in the analyses: GSM239041 (heads), GSM278695 (males), GSM278706 (females), GSM280085 (testes), GSM280082 (ovaries), GSM286604 (0-1 h embryos), GSM286605 (2-6 h embryos), GSM286607 (6-10 h embryos) and GSM272652 (S2). Reads have been mapped to $\mathrm{dm} 3$ genome assembly using megablast software and requiring perfect matching of the first 18 bases. Reads corresponding to annotated miRNA genes were extracted and their counts were used to infer miRNA expression profiles.

\section{Analysis of expression profiles of miRNAs}

To generate heatmaps and to calculate pair Pearson correlation coefficients $r$ for miRNA expression profiles, the relative frequencies of the miRs and miRs* abundances from the libraries were used. The Z-score normalization of miRNA expression profiles in heatmaps (rows) was applied. All operations and statistical calculations were performed by scripts in Perl and R languages.

\section{Prediction of cluster promoters}

The TSSs were predicted for the Drosophila genome (rel.5) by the McPromoter program [26] and were fetched from the program web-site (http://tools.genome. duke.edu/generegulation/McPromoter006). After mapping the TSSs on the $\mathrm{dm} 3$ genome assembly by the UCSC Genome Browser, the positions of cluster TSSs within up to $5 \mathrm{~Kb}$ upstream regions were evaluated by the UCSC Table Browser [39,40]. ChIP-seq data on pol II were fetched from modENCODE server http://www. modencode.org and mapped to $\mathrm{dm} 3$ using UCSC Genome Browser.

\section{Additional material}

Additional file 1: Tables S1, S2, S3, S4, S5, S6 and S7. Table S1 contains the brief summary of Drosophila miRNA clusters. Table S2 is the list of the analyzed libraries of sequenced small RNAs. Table S3 contains the relative frequencies of miRNAs occurring in the libraries. Table $\$ 4$ is the list of the putative TSSs of the miRNA clusters within up to $5 \mathrm{~Kb}$ upstream regions. Table S5 contains the probabilities to find TSS within inter-miRNA spaces in miRNA clusters. Tables $\mathrm{S} 6$ and $\mathrm{T7}$ are the results of the additional analysis of the expanded dataset.

Additional file 2: Heatmaps and correlation tables of clustered miRNA expression profiles.

Additional file 3: Genetic maps of the miRNA cluster TSSs.

\section{Acknowledgements}

The research was supported by funding from the "Molecular and Cell Biology" program of Russian Academy of Sciences to VAG.

\section{Author details}

'Institute of Molecular Genetics, Russian Academy of Sciences, Moscow, Russia. ${ }^{2}$ Hubrecht Institute, Royal Netherlands Academy of Arts and Sciences and University Medical Centre Utrecht, Utrecht, The Netherlands. ${ }^{3}$ Institute of Cytology and Genetics, Russian Academy of Sciences, Novosibirsk, Russia.

\section{Authors' contributions}

SSR designed the study, carried out the expression and promoter analyses. EB analyzed the small RNA libraries and helped to draft the manuscript. SSR and VAG wrote the manuscript. All authors read and approved the final manuscript.

Received: 7 December 2010 Accepted: 19 July 2011

Published: 19 July 2011

\section{References}

1. Bartel DP: MicroRNAs: genomics, biogenesis, mechanism, and function. Cell 2004, 116:281-297.

2. Filipowicz W, Bhattacharyya SN, Sonenberg N: Mechanisms of posttranscriptional regulation by microRNAs: are the answers in sight? Nat Rev Genet 2008, 9:102-114.

3. Guo H, Ingolia NT, Weissman JS, Bartel DP: Mammalian microRNAs predominantly act to decrease target mRNA levels. Nature 2010, 466:835-840.

4. Hendrickson DG, Hogan DJ, McCullough HL, Myers JW, Herschlag D, Ferrell JE, Brown PO: Concordant regulation of translation and mRNA abundance for hundreds of targets of a human microRNA. PLOS Biol 2009, 7:e1000238.

5. Okamura K, Phillips MD, Tyler DM, Duan H, Chou YT, Lai EC: The regulatory activity of microRNA* species has substantial influence on microRNA and 3' UTR evolution. Nat Struct Mol Biol 2008, 15:354-363.

6. Ro S, Park C, Young D, Sanders KM, Yan W: Tissue-dependent paired expression of miRNAs. Nucleic Acids Res 2007, 35:5944-5953.

7. Altuvia $Y$, Landgraf $P$, Lithwick $G$, Elefant $N$, Pfeffer $S$, Aravin A, Brownstein MJ, Tuschl T, Margalit H: Clustering and conservation patterns of human microRNAs. Nucleic Acids Res 2005, 33:2697-2706.

8. Merchan F, Boualem A, Crespi M, Frugier F: Plant polycistronic precursors containing non-homologous microRNAs target transcripts encoding functionally related proteins. Genome Biol 2009, 10:R136.

9. Baskerville S, Bartel DP: Microarray profiling of microRNAs reveals frequent coexpression with neighboring miRNAs and host genes. RNA 2005, 11:241-247.

10. Liang Y, Ridzon D, Wong $L$, Chen C: Characterization of microRNA expression profiles in normal human tissues. BMC Genomics 2007, 8:166.

11. Ruby JG, Stark A, Johnston WK, Kellis M, Bartel DP, Lai EC: Evolution, biogenesis, expression, and target predictions of a substantially expanded set of Drosophila microRNAs. Genome Res 2007, 17:1850-1864.

12. Yu J, Wang F, Yang GH, Wang FL, Ma YN, Du ZW, Zhang JW: Human microRNA clusters: genomic organization and expression profile in leukemia cell lines. Biochem Biophys Res Commun 2006, 349:59-68.

13. Kim YK, Yu J, Han TS, Park SY, Namkoong B, Kim DH, Hur K, Yoo MW, Lee HJ, Yang HK, Kim VN: Functional links between clustered microRNAs: suppression of cell-cycle inhibitors by microRNA clusters in gastric cancer. Nucleic Acids Res 2009, 37:1672-1681.

14. $\mathrm{Xu} \mathrm{J}$, Wong $\mathrm{C}:$ A computational screen for mouse signaling pathways targeted by microRNA clusters. RNA 2008, 14:1276-1283.

15. Biemar F, Zinzen R, Ronshaugen M, Sementchenko V, Manak JR, Levine MS: Spatial regulation of microRNA gene expression in the Drosophila embryo. Proc Natl Acad Sci USA 2005, 102:15907-15911.

16. Houbaviy HB, Dennis L, Jaenisch R, Sharp PA: Characterization of a highly variable eutherian microRNA gene. RNA 2005, 11:1245-1257.

17. Lee Y, Kim M, Han J, Yeom KH, Lee S, Baek SH, Kim VN: MicroRNA genes are transcribed by RNA polymerase II. EMBO J 2004, 23:4051-4060.

18. Heo I, Joo C, Cho J, Ha M, Han J, Kim VN: Lin28 mediates the terminal uridylation of let-7 precursor MicroRNA. Mol Cell 2008, 32:276-284. 
19. Newman MA, Thomson JM, Hammond SM: Lin-28 interaction with the Let-7 precursor loop mediates regulated microRNA processing. RNA 2008, 14:1539-1549.

20. Piskounova E, Viswanathan SR, Janas M, LaPierre RJ, Daley GQ, Sliz P, Gregory Rl: Determinants of microRNA processing inhibition by the developmentally regulated RNA-binding protein Lin28. J Biol Chem 2008, 283:21310-21314.

21. Rybak A, Fuchs H, Smirnova L, Brandt C, Pohl EE, Nitsch R, Wulczyn FG: A feedback loop comprising lin-28 and let-7 controls pre-let-7 maturation during neural stem-cell commitment. Nat Cell Biol 2008, 10:987-993.

22. Sokol NS, Xu P, Jan YN, Ambros V: Drosophila let-7 microRNA is required for remodeling of the neuromusculature during metamorphosis. Genes Dev 2008, 22:1591-1596.

23. Graveley BR, Brooks AN, Carlson JW, Duff MO, Landolin JM, Yang L, Artieri CG, van Baren MJ, Boley N, Booth BW, Brown JB, Cherbas L, Davis CA, Dobin A, Li R, Lin W, Malone JH, Mattiuzzo NR, Miller D, Sturgill D, Tuch BB, Zaleski C, Zhang D, Blanchette M, Dudoit S, Eads B, Green RE, Hammonds A, Jiang L, Kapranov P, Langton L, Perrimon N, Sandler JE, Wan KH, Willingham A, Zhang Y, Zou Y, Andrews J, Bickel PJ, Brenner SE, Brent MR, Cherbas P, Gingeras TR, Hoskins RA, Kaufman TC, Oliver B, Celniker SE: The developmental transcriptome of Drosophila melanogaster. Nature 2010.

24. Zhou X, Ruan J, Wang G, Zhang W: Characterization and identification of microRNA core promoters in four model species. PLoS Comput Biol 2007, 3:e37.

25. FitzGerald PC, Sturgill D, Shyakhtenko A, Oliver B, Vinson C: Comparative genomics of Drosophila and human core promoters. Genome Biol 2006, 7:R53

26. Ohler U: Identification of core promoter modules in Drosophila and their application in accurate transcription start site prediction. Nucleic Acids Res 2006, 34:5943-5950.

27. Rach EA, Yuan HY, Majoros WH, Tomancak P, Ohler U: Motif composition, conservation and condition-specificity of single and alternative transcription start sites in the Drosophila genome. Genome Biol 2009, 10: R73.

28. Fujita $\mathrm{S}, \mathrm{Iba} \mathrm{H}$ : Putative promoter regions of miRNA genes involved in evolutionarily conserved regulatory systems among vertebrates. Bioinformatics 2008, 24:303-308.

29. Saini HK, Griffiths-Jones S, Enright AJ: Genomic analysis of human microRNA transcripts. Proc Natl Acad Sci USA 2007, 104:17719-17724.

30. Berezikov E, Robine N, Samsonova A, Westholm JO, Naqvi A, Hung JH, Okamura K, Dai Q, Bortolamiol-Becet D, Martin R, Zhao Y, Zamore PD, Hannon GJ, Marra MA, Weng Z, Perrimon N, Lai EC: Deep annotation of Drosophila melanogaster microRNAs yields insights into their processing, modification, and emergence. Genome Res 2011, 21:203-215.

31. Guil S, Caceres JF: The multifunctional RNA-binding protein hnRNP A1 is required for processing of miR-18a. Nat Struct Mol Biol 2007, 14:591-596.

32. Buck AH, Perot J, Chisholm MA, Kumar DS, Tuddenham L, Cognat V, Marcinowski L, Dolken L, Pfeffer S: Post-transcriptional regulation of miR27 in murine cytomegalovirus infection. RNA 2010, 16:307-315.

33. Linsen $S E$, de WE, Janssens $G$, Heater $S$, Chapman L, Parkin RK, Fritz B, Wyman SK, de BE, Voest EE, Kuersten S, Tewari M, Cuppen E: Limitations and possibilities of small RNA digital gene expression profiling. Nat Methods 2009, 6:474-476.

34. Pfeffer S, Sewer A, Lagos-Quintana M, Sheridan R, Sander C, Grasser FA, van Dyk LF, Ho CK, Shuman S, Chien M, Russo JJ, Ju J, Randall G, Lindenbach BD, Rice CM, Simon V, Ho DD, Zavolan M, Tuschl T: Identification of microRNAs of the herpesvirus family. Nat Methods 2005, 2:269-276.

35. McCormick KP, Willmann MR, Meyers BC: Experimental design, preprocessing, normalization and differential expression analysis of small RNA sequencing experiments. Silence 2011, 2:2.

36. Munafo DB, Robb GB: Optimization of enzymatic reaction conditions for generating representative pools of cDNA from small RNA. RNA 2010, 16:2537-2552.

37. Krol J, Loedige I, Filipowicz W: The widespread regulation of microRNA biogenesis, function and decay. Nat Rev Genet 2010, 11:597-610

38. Slezak-Prochazka I, Durmus S, Kroesen BJ, van den Berg A: MicroRNAs, macrocontrol: regulation of miRNA processing. RNA 2010, 16:1087-1095.
39. Karolchik D, Hinrichs AS, Furey TS, Roskin KM, Sugnet CW, Haussler D, Kent WJ: The UCSC Table Browser data retrieval tool. Nucleic Acids Res 2004, 32:D493-D496.

40. Kent WJ, Sugnet CW, Furey TS, Roskin KM, Pringle TH, Zahler AM, Haussler D: The human genome browser at UCSC. Genome Res 2002, 12:996-1006.

doi:10.1186/1471-2164-12-371

Cite this article as: Ryazansky et al:: Evidence for post-transcriptional regulation of clustered microRNAs in Drosophila. BMC Genomics 2011 $12: 371$.

\section{Submit your next manuscript to BioMed Central and take full advantage of:}

- Convenient online submission

- Thorough peer review

- No space constraints or color figure charges

- Immediate publication on acceptance

- Inclusion in PubMed, CAS, Scopus and Google Scholar

- Research which is freely available for redistribution

Submit your manuscript at www.biomedcentral.com/submit
C Biomed Central 